\title{
Numerical simulation of flux avalanches in type-II superconducting thin films under transient AC magnetic fields
}

\author{
Ze Jing ${ }^{1,2}$ a) and Mark D. Ainslie ${ }^{2}$ \\ ${ }^{1}$ Research Center of Applied Mechanics, School of Mechano-Electronic Engineering, Xidian \\ University, Xian, Shaanxi 710071, People's Republic of China \\ ${ }^{2}$ Department of Engineering, University of Cambridge, Trumpington Street, Cambridge CB2 \\ $1 P Z, U K$
}

\begin{abstract}
Flux avalanches induced from thermomagnetic instability are crucial challenges for the application of superconducting thin film devices. In this paper, flux avalanches in a type-II superconducting thin film exposed to a transient $\mathrm{AC}$ magnetic field are numerically simulated by solving the coupled nonlinear Maxwell's equations and the heat diffusion equation based on the fast Fourier transform (FFT) method. The dependence of the threshold magnetic field on the ambient temperature, film thickness and magnetic field ramp rate are obtained through these numerical simulations, which show good agreement with experimental results. A linear increase in the threshold field as the film thickness increases and a nonmonotonic increase in the threshold field as the ambient temperature increases have also been found. Our numerical results demonstrate that the threshold field decreases exponentially as the ramp rate increases. Flux avalanche patterns observed in magneto-optical imaging (MOI) measurements for a film exposed to an AC magnetic field are reproduced. The hysteresis magnetization and maximum temperature jump curves are also illustrated. We find that fingering instability plays an important role in the thermomagnetic response of superconducting thin films under transient AC magnetic fields, especially for high magnetic field ramp rates.
\end{abstract}

a) Corresponding author Email: zejing@xidian.edu.cn 


\section{Introduction}

Superconducting (SC) thin films are widely used in many application areas, such as superconducting quantum interference devices [1], microwave resonators [2], singlephoton optical detectors [3, 4], Josephson junctions [5] and so on. The dendritic flux avalanche is one of the most commonly encountered challenges in the application of superconducting thin film devices. When a flux avalanche occurs, the normally smooth magnetic field penetration will be interrupted by the sudden rush in or out of large numbers of vortices, which form dendritic structures in the superconducting film $[6,7]$.

Flux avalanches originate from the thermomagnetic instability of superconducting thin films [8]. Above the lower critical magnetic field $H_{c l}$, magnetic flux penetrates into the type-II superconductor in the form of vortices (or flux lines) [9]. Any thermal or electromagnetic fluctuation will weaken the pinning of some vortices and initiate their motion [10]. Motion of the vortices dissipates energy and locally heats the superconductor. If the heat generated cannot be removed from the hotspot area in a timely manner, the pinning reduces even further which facilitates more vortices to move, and a positive feedback loop develops [11]. Thus, a dendritic flux avalanche is a result of competition between the dissipative vortex motion, thermal diffusion within the superconductor and heat transfer to the surrounding coolant, combined with nonlocal and nonlinear electrodynamics. Flux avalanches are usually considered unfavorable to the application of superconductors: 1) the suppression of critical current density in the hotspot area decreases current carrying capacity [12]; 2) the entry or exit of large numbers of vortices causes flux jumps and generates noise in superconducting devices [13]; 3) a sharp rise in the local temperature in the hotspot area induces a large thermal strain, and can cause irreversible damage and melting of the SC film when the temperature rises high enough $[14,15]$. On the other hand, flux avalanches can be exploited to design flux injectors to allow for controlled injections of magnetic flux into superconducting films [16]; and flux jumps can be exploited for pulsed field magnetization of bulk superconductors $[17,18]$. Thus, investigating flux avalanches in superconducting thin films is of significant importance from both theoretical and 
practical applications perspectives.

Experiments using magneto-optical imaging (MOI) have revealed that flux avalanches take place in numerous superconducting materials like $\mathrm{YBa}_{2} \mathrm{Cu}_{3} \mathrm{O}_{7-\delta}[14$, 19-21], $\mathrm{MgB}_{2}$ [22, 23], $\mathrm{Nb}_{3} \mathrm{Sn}$ [24-26], $\mathrm{YNi}_{2} \mathrm{~B}_{2} \mathrm{C}$ [27], $\mathrm{Pb}$ [28-30], $\mathrm{NbN}$ [31], and so on. Except for the direct observations using MOI and other techniques to image the dendritic avalanche patterns, many theoretical efforts have been made to understand the thermomagnetic instability. Denisov and Dvash et al. [32-34] investigated the threshold condition for the thermomagnetic instability through linear stability analysis of small perturbations. Rakhmanov et al. [35] have obtained criteria for the onset of thermomagnetic instability of superconducting thin films that produced finger patterns. Aranson et al. [36-38] investigated the dendritic flux avalanches and the nonlocal electrodynamics of an infinitely long superconducting slab by numerically solving Maxwell's equations coupled with the heat diffusion equation. Zhou et al. [39, 40] have made significant contributions to the numerical simulations of flux jumps of superconducting slabs and bulks. To reveal the evolution of flux avalanches from its nucleation stage to the fully developed dendritic avalanche stage, Vestgården et al. [7, 41-44] proposed a fast Fourier transformation (FFT) based iteration scheme to simulate the nonlocal electrodynamics of a finite-sized superconducting film. The scheme has been widely used to simulate the electrodynamics of various SC films under different circumstances, and the simulated flux avalanche patterns are strikingly similar to experimental observations [45-48]. However, simulations on the experimentally observed dendritic instability of superconducting thin films during the flux entrance and exit process for a transient magnetic field especially during an AC cycle $[49,50]$ have not been reported yet.

In this paper, we report an investigation on the numerical simulations of flux avalanches in superconducting thin films exposed to transient AC magnetic fields. The magnetic flux distribution and the temperature evolution are simulated by solving coupled nonlocal electrodynamic equations and the thermal diffusion equation. We considered the effect of ambient temperature, film thickness and magnetic field ramp 
rate on the avalanche behavior. The threshold magnetic field is also obtained through these numerical simulations. In section 2, we briefly introduce the theoretical formalisms used to characterize the flux avalanche behavior of SC films. The evolution of the magnetic flux and the temperature distribution in the superconducting film, as well as the threshold magnetic field for the avalanches are presented and discussed in Section 3. Finally, the conclusions are summarized in Section 4.

\section{Numerical Modelling}

The nonlocal electrodynamics of type-II superconducting thin films are governed by Maxwell's equations:

$$
\nabla \times \boldsymbol{E}=-\frac{\partial \boldsymbol{B}}{\partial t}, \nabla \cdot \boldsymbol{B}=0, \text { and } \nabla \times \boldsymbol{H}=\boldsymbol{J} \delta(z),
$$

in which $\boldsymbol{E}$ is the electric field, $\boldsymbol{J}$ is the sheet current density, $\boldsymbol{H}$ is the magnetic field strength and relates to the magnetic field by $\boldsymbol{B}=\mu_{0} \boldsymbol{H}$, and $\delta(z)$ is the Dirac delta function. The distribution of temperature is described by the heat diffusion equation:

$$
d c \frac{\partial T}{\partial t}=d \nabla \cdot(\kappa \nabla T)-h\left(T-T_{0}\right)+\boldsymbol{J} \cdot \boldsymbol{E}
$$

where $\kappa$ is the thermal conductivity, $c$ is the specific heat, $T_{0}$ is the ambient temperature, $h$ is the heat transfer coefficient, and $d$ is the thickness of the SC film. $\kappa, c$, and $h$ all vary with the temperature, $T$. The nonlinear voltage-current relationship which describes the sharp depinning of vortices in the superconductor is given by [43, 51]:

$$
\begin{aligned}
& \boldsymbol{E}=\rho_{f}(J) \boldsymbol{J} / d, \\
& \rho_{f}(\mathrm{~J})=\left\{\begin{array}{lr}
\rho_{0}\left(J / J_{c}\right)^{n-1}, & J \leq J_{c}, T \leq T_{c}, \\
\rho_{\text {normal }}, & \text { otherwise. }
\end{array}\right.
\end{aligned}
$$

where $n$ is the flux creep exponent, and it is assumed that $n=90\left(\frac{T_{c}}{T}\right)-50[35,52] . \rho_{0}$ is a resistivity constant for the flux creep state, and $\rho_{\text {nornal }}$ is the normal state resistivity. $T$ is the local temperature, $T_{c}$ is the critical temperature, and $J_{c}$ is the critical sheet current density which takes the following form:

$$
J_{c}=J_{c 0}(B)\left[1-\left(T / T_{c}\right)\right]
$$

where the magnetic field dependence of the critical current density is taken into 
consideration, and Kim's model $[53] J_{c 0}(B)=J_{c 0} /\left(1+B / B_{0}\right)$ is adopted.

Here, we consider a square-shaped superconducting thin film of length and width $L=W=2 a$, and thickness $d$ deposited onto a substrate (as shown in figure 1(a)). The film is zero-field-cooled below the critical temperature $T_{c}$, and subjected to an AC magnetic field perpendicular to the film plane at the ambient temperature $T_{0}$. The waveform of the fast-cycling transient $\mathrm{AC}$ magnetic field is triangular, as shown in figure 1(b). The fast Fourier transform based iteration algorithm proposed by Vestgården et al $[45,48,54]$ is adopted to simulate the flux avalanche behavior of the superconducting film. A numerical program coded with Matlab is implemented for this purpose. The method of lines is used to solve the coupled nonlinear equations (1) and (2). The simulation domain is discretized with $256 \times 256$ equidistant grids, and the time is integrated as initial value ordinary differential equations. The flux penetration process starts from a zero-field-cooled state with no flux trapped in the superconducting film under a uniform temperature $T_{0}$. The material parameters of the superconducting thin film assumed in the simulations are the typical parameters of $\mathrm{Nb}$ film $[35,52]$ with $\rho_{0}$ $=\rho_{\text {normal }}=6 \times 10^{-9} \Omega \cdot \mathrm{m}, T_{c}=9.2 \mathrm{~K}, B_{0}=100 \mathrm{mT}, d=50 \mathrm{~nm}$, and $a=1.5 \mathrm{~mm}$. The heat capacity of the $\mathrm{Nb}$ film is assumed to be $c=2 \times 10^{4}\left(T / T_{c}\right)^{3} \mathrm{~J} / \mathrm{m}^{3} \cdot \mathrm{K}$, the thermal conductivity $\kappa=20\left(T / T_{c}\right)^{3} \mathrm{~W} / \mathrm{m} \cdot \mathrm{K}$, and the coefficient of heat transfer between the superconducting film and the substrate is assumed to be $h=2 \times 10^{3}\left(T / T_{c}\right)^{3} \mathrm{~W} / \mathrm{m}^{2} \cdot \mathrm{K}$. We assume that the substrate is an electrical insulator and there is no electric current in the substrate.

\section{Numerical Results and Discussions}

From experimental measurements and theoretical analysis, thermomagnetic instability occurs in thin film superconductors above a certain threshold magnetic field, and this threshold field depends on the ambient temperature $T_{0}$, the film thickness $d$, and the ramp rate of external field, and so on. Here, we present our numerical simulation results on the threshold magnetic field and the avalanche characteristics of the $\mathrm{Nb}$ thin film under transient AC magnetic fields. 


\subsection{Threshold magnetic field for thermomagnetic instability}

According to the analytical analysis in [55], when the main mechanism for suppression of instability is lateral heat diffusion under small magnetic field $H_{a}$, the threshold magnetic field, $H_{t h, \kappa}$, at which the thermomagnetic instability event first takes place in the superconducting film becomes

$$
H_{t h, \kappa}=\frac{d j_{c}}{\pi}\left(\frac{\pi^{2} \kappa T^{*}}{n w^{3} j_{c} \mu_{0} \dot{H}_{a}}\right)^{1 / 5}
$$

where $T^{*}=\left|\partial \ln j_{c} / \partial T\right|^{-1}$ and the parameters $j_{c}, \kappa$ and $n$ are temperature dependent. Regarding the adiabatic condition, when the instability is prevented only by the heat capacity of the superconductor, the threshold magnetic field can be given as [56]

$$
H_{t h, c}=\sqrt{\frac{2}{\pi} \frac{c T^{*}}{\mu_{0}} \frac{d}{w}}
$$

\section{A. Effect of ambient temperature}

Figure 2 shows the magnetic flux patterns of a $50 \mathrm{~nm}$ thick $\mathrm{Nb}$ film exposed to a magnetic field ramped from 0 to $2 \mathrm{mT}$ at a ramp rate of $1000 \mathrm{~T} / \mathrm{s}$ for different ambient temperatures. The bright color indicates the positive flux, while the dark one indicates negative flux. Initially, most of the superconducting film is in the Meissner state. As the field increases, the magnetic flux penetrates gradually from the edges. When the applied field reaches its threshold value, the smooth flux penetration will be interrupted by flux avalanches, starting preferentially from the central part along the edges. A further increase in the magnetic field results in more avalanches, entering one by one. At the early stage of penetration, the flux avalanche patterns show finger-like structures. If the applied field continues to increase, pronounced dendritic flux avalanches penetrate into the film. Each finger-like or branch structure shown in figure 2 indicates a flux avalanche event. It should be obvious that the flux avalanche behavior changes significantly as the ambient temperature changes. As seen in figure $2\left(\mathrm{a}_{1}\right)-\left(\mathrm{d}_{1}\right)$, under an external magnetic field ramped to the same value, more flux avalanche events occur in the SC film at lower ambient temperatures. Comparing the avalanche patterns at $3 \mathrm{~K}, 4$ $\mathrm{K}, 5 \mathrm{~K}$ and $6 \mathrm{~K}$, it is also found that a transition from finger-like avalanche patterns to 
more dendritic flux avalanches occurs when the ambient temperature increases. Similar results have been found experimentally in $\mathrm{Nb}$ films, where the $\mathrm{SC}$ film also shows a nonmonotonic response to the increasing temperature [49]. The dendritic flux avalanche is a result of flux motion and the local increase in temperature. The critical current density $J_{c}(T)$ is a measure of the resistance to the flux penetration, which decreases as the temperature increases. At higher temperatures, the magnetic flux more easily enters the film which reduces the local heating. Thus, the threshold magnetic field becomes higher as the ambient temperature increases. In addition, it is also seen that the avalanche morphology exhibits more "diffused thicker" branches at higher temperatures. This is explained by the fact that width of the avalanche branch is mainly determined by the thermal diffusion perpendicular to the avalanche paths, and the thermal diffusion coefficient $\kappa$ increases as the ambient temperature increases.

The temperature dependence of the threshold magnetic field for the $50 \mathrm{~nm}$ thick $\mathrm{Nb}$ film exposed to a magnetic field ramped from zero field at a rate of $1000 \mathrm{~T} / \mathrm{s}$ is shown in figure 3 . The black squares in figure 3 represent our numerical simulation results. The dashed and dotted curves are the threshold fields obtained analytically based on the assumption that the main mechanism for suppression of the instability is heat capacity (adiabatic) and lateral heat diffusion, respectively [55] (see equations (5) and (6)). The red dots are the experimental results from [57] multiplied by a factor of 0.02 , which is obtained from the theoretical expression (5), since we used a much higher magnetic field ramp rate in the simulation which is different from reference [57]. In addition, one of the main focuses of this work is to investigate the effect of AC magnetic field on the flux avalanches. We used the specific geometric parameters to obtain results comparable with the experimental results in references [49] and [50]. Hence, we adopted the scaling expression (5) to remedy this discrepancy from the experiments in reference [57]. According to the analytical expression (5), the threshold magnetic field $H_{t h, \kappa} \propto w^{-3 / 5} d^{3 / 5} \dot{H}_{a}^{-1 / 5}$, in which the thickness dependence of the critical current density $\left(j_{\mathrm{c}} \propto d^{-0.5}\right)$ is taken into consideration [58]. The ratio between the characteristic lengths of the film in our simulations and the experiments is $r_{w}=1.5 \mathrm{~mm} / 4.5 \mathrm{~mm}=1 / 3$. The 
ratio between the thicknesses is $r_{d}=50 \mathrm{~nm} / 0.5 \mu \mathrm{m}=0.1$, and the ratio between the field ramp rates is $r_{\text {Hat }}=(1000 \mathrm{~T} / \mathrm{s}) /(0.1 \mathrm{mT} / \mathrm{s})=10^{7}$. Thus, the ratio of the threshold field between our numerical simulations and the experiments is $H_{t h, \kappa}^{s} / H_{t h, \kappa}^{e}=\left(r_{w}\right)^{-}$ ${ }^{3 / 5}\left(r_{d}\right)^{3 / 5}\left(r_{\text {Hat }}\right)^{-1 / 5} \approx 0.02$. It is shown in figure 3 that the numerical simulation results are in good agreement with the experimental results. Comparing the numerical simulation results with analytical results, we can see that most of the numerical simulation and experimental results fall between the two curves. This is reasonable because both heat capacity and heat diffusion play significant roles in the thermomagnetic instability of superconductors. Our results show a sharp increase in the threshold magnetic field as the ambient temperature increases.

\section{B. Effect of SC film thickness}

In addition, film thickness has a significant effect on the thermomagnetic instability of thin film superconductors. In figure 4, we present the simulated flux avalanche patterns in the $\mathrm{Nb}$ film with different thicknesses. The film is exposed to a magnetic field ramped from zero to $2 \mathrm{mT}$ at a rate of $50 \mathrm{~T} / \mathrm{s}(\mathrm{f}=6.25 \mathrm{kHz})$ at $3 \mathrm{~K}$ ambient temperature. As shown in figure 4, avalanches in the thin film show a fingerlike penetration profile, which is in qualitative agreement with the experimental MOI results [49]. For the thicker films, fewer avalanche events occur for the same external magnetic field and ambient temperature than for the thinner ones, and there is also a transition in the avalanche morphology from the branch tree-like to the straight fingerlike pattern.

The thickness dependence of the threshold magnetic field on the film thickness is shown in figure 5 . The dark gray squares are the numerical simulation results, where each data point represents the threshold magnetic field of the SC film for different thicknesses. The red dots indicate the experimental results taken from [59], where the geometry of the $\mathrm{Nb}$ film and other experimental conditions are similar to our simulations. The numerical simulation results show good agreement with the experimental ones. The threshold magnetic field follows a linear dependence on the film thickness, for both the numerical simulations and experiments. As expected, the 
thicker the SC film, the higher the threshold magnetic field.

\section{Effect of magnetic field ramp rate}

The influence of the external magnetic field ramp rate on flux avalanche patterns in the $\mathrm{Nb}$ thin film is shown in figure 6. Figures $6\left(\mathrm{a}_{1}\right)-\left(\mathrm{a}_{4}\right),\left(\mathrm{b}_{1}\right)-\left(\mathrm{b}_{4}\right)$ and $\left(\mathrm{c}_{1}\right)-\left(\mathrm{c}_{4}\right)$ show the numerically simulated magnetic flux distribution within the $\mathrm{Nb}$ film, when the external magnetic field is ramped from zero to $2 \mathrm{mT}$ at the rates of $8 \mathrm{~T} / \mathrm{s}, 100 \mathrm{~T} / \mathrm{s}$ and $1000 \mathrm{~T} / \mathrm{s}$, respectively. As shown in figure 6(a1)-(a4), when the magnetic field ramp rate is low $(8 \mathrm{~T} / \mathrm{s})$, the flux smoothly penetrates into the SC film initially. If the external magnetic field increases further, giant dendritic flux avalanches penetrate into the film (see figure 6( $\mathrm{a}_{3}$ ) and (a4), for example). For higher ramp rates, fingering flux avalanches penetrate from the edges, and the number of avalanche events increases, while fewer giant dendritic avalanches occur in the film. Hence, when the external magnetic field ramp rate increases further, there is a transition from the branched dendritic flux avalanches to the finger-like dominated avalanche patterns. As predicted in [37], based on the linear perturbation analysis of heat diffusion and Maxwell's equations, fingering instability arises when the magnetic field ramp rate is so large that the magnetic flux diffusion proceeds much faster than the heat diffusion. Microscopically, this may also be explained by the fact that under such an extremely high magnetic field ramp rate, vortices penetrate into the SC film with ultrafast velocities. The initially penetrated vortices form heated vortex channels, subsequent vortices penetrate along these channels, and do not have enough time to spread away from the channel, hence there is no bifurcation in the avalanche channels [60].

The threshold magnetic field for the flux avalanches of the SC film under different external magnetic field ramp rate are shown in figure 7 . The red squares indicate the numerical simulation results, each representing the magnetic field at which the first avalanche event occurs. The solid blue line shows the threshold magnetic field as a function of magnetic field ramp rate obtained from analytical expression (5). One can see that the threshold magnetic field decreases exponentially as the external magnetic field ramp rate increases. The numerical simulation results agree quantitatively well 
with the analytical results. In the high magnetic field ramp rate region, large electric field $E\left(\sim \dot{H}_{a} H / j_{c}\right)$ is induced in the film. When the electric field is larger than a certain critical value, nonuniform fingering instability develops (as the inset images show). According to the theoretical perturbation analysis, threshold magnetic field for the fingering instability is given as $H_{\text {fing }} \sim \pi / 2 \sqrt{\kappa T^{*} j_{c} / E}[37,56]$, which is usually much smaller than that for dendritic flux avalanches.

\subsection{Flux avalanches in an SC film under $\mathrm{AC}$ magnetic fields}

Since magnetic field variation (both the ramp rate and magnitude) is crucial to the occurrence of flux avalanches, the thermomagnetic instability will be enhanced when the SC film is exposed to an AC magnetic field. Here, we consider a $50 \mathrm{~nm}$ thick $\mathrm{Nb}$ film under an AC triangular cyclic field with a ramp rate of $1000 \mathrm{~T} / \mathrm{s}$ at $3 \mathrm{~K}$. Figures 8(a) and (b) present the hysteresis curve for the magnetization jumps and the maximum temperature evolution in the film, respectively. The maximum temperature is defined as the maximum of the local temperature in the superconducting thin film. The inset images 1 to 5 in figure $8(a)$ are the flux avalanche patterns of the film when: the field increased from zero to $+0.4 \mathrm{mT}$, decreased back to zero and to $-0.4 \mathrm{mT}$, and then increased again to zero and to $+0.4 \mathrm{mT}$, respectively. The images correspond to the arrows on the hysteresis magnetization curve. It can be seen in figure 8(b) that the first avalanche event takes place at about $\mu_{0} H_{a}=0.112 \mathrm{mT}$ and more flux avalanches penetrate into the film as the external magnetic field increases further. When the magnetic field decreases, reversed vortices penetrate into the film through the same tracks, and antiflux avalanches occur during this process in the form of finger-type patterns (see image 2). Flux and antiflux coexist within the SC film when the external magnetic field deceased to zero. More antiflux penetrate into the film as the external magnetic field decreases further, and the antiflux avalanche dominates in the film after the magnetic field eventually decreased to its minimum $-0.4 \mathrm{mT}$ (see image 3 ). As the external magnetic field increases again, positive flux penetrates into the film in the form of finger-like avalanches almost along the same tracks of the antiflux avalanches. Our 
numerically simulated flux avalanche patterns are in good agreement with the magnetooptical visualization shown in figure 3 from [49]. In figure 8(b), we present the maximum temperature evolution within the film during the $\mathrm{AC}$ magnetic field. Compared with the simulation results previously reported for $\mathrm{MgB}_{2}$ thin film $[46,47$, 54], the temperature of the $\mathrm{Nb}$ thin film continuously increases as the external magnetic field varies. This may be attributed to the fact that under such transient magnetic fields there is not enough time for the heat generated during an avalanche event to transfer to the surrounding coolant before subsequent avalanche events occur.

In addition, we have also investigated the effects of magnetic field ramp rates on the flux avalanche behavior. Figure 9 presents the flux avalanche patterns of the film exposed to a magnetic field ramped from zero to $+2 \mathrm{mT}$, then decreased back to zero and to $-2 \mathrm{mT}$, followed by increasing to zero and $+2 \mathrm{mT}$. Figures $9\left(\mathrm{a}_{1}\right)-\left(\mathrm{a}_{5}\right)$ and figures 9(b1)-(bs) illustrate the magnetic flux patterns of the SC film exposed to the magnetic field at the ramp rate of $8 \mathrm{~T} / \mathrm{s}$ and $1000 \mathrm{~T} / \mathrm{s}$, respectively. As shown in figure 9 , the morphology of the flux avalanche pattern reveals a quite disordered mixture of flux and antiflux regions while the film is exposed to the AC magnetic field. As shown in figure 9 (a 1 )-(a5), giant dendritic flux avalanches penetrate into the SC film after the magnetic field increased to $2 \mathrm{mT}$ at the rate of $8 \mathrm{~T} / \mathrm{s}$. When the external magnetic field ramps down to zero, antiflux avalanches penetrate into the film along the tracks already formed when the field was increasing. From figure $9\left(a_{2}\right)$, we can see that once avalanches penetrate into the SC film, they remain "frozen" during the subsequent decrease of the applied field. As the applied field decreases further to $-2 \mathrm{mT}$ (see figure 9(a3)), more antiflux avalanches penetrate into the film, and the magnetic flux distribution shows mosaic patterns. When the applied field increases back to zero and $+2 \mathrm{mT}$ again, flux avalanches penetrate into the film and overlap the previously formed avalanche tracks (see figure $9\left(a_{4}\right)$ and (as)). Moreover, the magnetic field ramp rate has a significant effect on the flux distribution and avalanche patterns. From the avalanche patterns shown in figure $9\left(b_{1}\right)-\left(b_{5}\right)$, we can see that straight avalanche channels penetrate into the SC film when the ramp rate is $1000 \mathrm{~T} / \mathrm{s}$. 
In figure 10, we present (a) the hysteresis magnetization jumping curves and (b) the maximum temperature evolution of the SC film subjected to AC magnetic fields of ramp rates $8 \mathrm{~T} / \mathrm{s}, 100 \mathrm{~T} / \mathrm{s}$ and $1000 \mathrm{~T} / \mathrm{s}$, respectively. It is shown that the average magnetization as well as the maximum temperature jump size become smaller as the magnetic field ramp rate increases. For the low $\operatorname{ramp}(8 \mathrm{~T} / \mathrm{s})$, the temperature can return back to the ambient temperature after an avalanche event. However, as the magnetic field ramp rate increases, the temperature increases continuously as more and more avalanche events occurs in the film. From the maximum temperature evolution in figure 10(b), we can infer that the temperature within the $\mathrm{Nb}$ film increases continuously as the $\mathrm{AC}$ cycle proceeds for high ramp rates.

\section{Conclusions}

In conclusion, a coupled thermomagnetic formulation has been proposed to numerically simulate the flux avalanche behavior of $\mathrm{Nb}$ thin films. The effects of ambient temperature, magnetic field ramp rate and film thickness on the thermomagnetic instability of the $\mathrm{Nb}$ thin film are investigated in detail. The dependence of the threshold magnetic field on the ambient temperature, the magnetic field ramp rate and the film thickness are obtained from the numerical simulations, and have been compared with both experimental results and analytical expressions based on adiabatic and thermal diffusion assumptions. The numerical results show good agreement with both. Furthermore, our numerical results demonstrate that both heat capacity and lateral thermal diffusion play important roles in the suppression of thermomagnetic instability in $\mathrm{Nb}$ thin films. The simulation results show the transition from dendritic to finger-like avalanches when the magnetic field ramp rate increased higher than certain value. The experimentally observed nonmonotonic response of the thermomagnetic instability has been verified in this paper. Furthermore, flux avalanches triggered by a transient AC magnetic field have also been investigated by numerical simulations. Flux avalanche patterns observed in MOI measurements are reproduced, and the hysteresis magnetization jump curves are also obtained. The simulation results 
show that under AC magnetic fields most avalanche dendrites are reused during the subsequent flux entrance or exit process. The flux avalanches patterns transit from dendritic tree-like to the finger-like ones as the magnetic field ramp rate of the transient AC magnetic field increases. The hysteresis magnetization loops show noticeable noisy jumping response during both the increasing and decreasing process of the external magnetic field. The average magnetization and the local temperature jump size gets smaller as the ramp rate of the AC magnetic field increases. Our results show that the superconducting thin films are more vulnerable to flux avalanches under transient AC magnetic field, which need careful consideration in designing superconducting thin film devices.

\section{Acknowledgements}

ZJ acknowledge the support from the National Natural Science Foundation of China (Nos. 11602185 and 11972271), Natural Science Basic Research Plan in Shaanxi Province of China (Grant No. 2018JQ1013) and the financial support from China Scholarship Council. MA would like to acknowledge financial support from an Engineering and Physical Sciences Research Council (EPSRC) Early Career Fellowship, EP/P020313/1. All data are provided in full in the results section of this paper.

\section{References}

[1] Kleiner R, Koelle D, Ludwig F and Clarke J 2004 Superconducting quantum interference devices: State of the art and applications Proc. IEEE 92 1534-48

[2] Vissers M R, Weides M P, Kline J S, Sandberg M and Pappas D P 2012 Identifying capacitive and inductive loss in lumped element superconducting hybrid titanium nitride/aluminum resonators Appl. Phys. Lett. 101022601

[3] Natarajan C M, Tanner M G and Hadfield R H 2012 Superconducting nanowire single-photon detectors: physics and applications Supercond. Sci. Tech. 25063001

[4] Pracht U S, Heintze E, Clauss C, Hafner D, Bek R, Werner D, Gelhorn S, Scheffler

M, Dressel M, Sherman D, Gorshunov B, Il'in K S, Henrich D and Siegel M 2013 Electrodynamics of the Superconducting State in Ultra-Thin Films at THz Frequencies IEEE Trans. Thz Sci. Techn. 3 269-80 
[5] Gross R, Alff L, Beck A, Froehlich O M, Koelle D and Marx A 1997 Physics and technology of high temperature superconducting Josephson junctions IEEE Trans. Appl. Supercond. 7 2929-35

[6] Altshuler E and Johansen T H 2004 Colloquium: Experiments in vortex avalanches Rev. Mod. Phys. 76 471-87

[7] Vestgården J I, Johansen T H and Galperin Y M 2018 Nucleation and propagation of thermomagnetic avalanches in thin-film superconductors (Review Article) Low Temp. Phys. 44 460-76

[8] Wipf S L 1991 Review of Stability in High-Temperature Superconductors with Emphasis on Flux Jumping Cryogenics 31 936-48

[9] Tinkham M 2004 Introduction to Superconductivity: Courier Corporation [10]Anderson P W and Kim Y B 1964 Hard Superconductivity: Theory of the Motion of Abrikosov Flux Lines Rev. Mod. Phys. 36 39-43

[11]Mints R G and Rakhmanov A L 1981 Critical State Stability in Type-II Superconductors and Superconducting-Normal-Metal Composites Rev. Mod. Phys. 53 551-92

[12]Zhao Z W, Li S L, Ni Y M, Yang H P, Liu Z Y, Wen H H, Kang W N, Kim H J, Choi E M and Lee S I 2002 Suppression of superconducting critical current density by small flux jumps in $\mathrm{MgB}_{2}$ thin films Phys. Rev. B $\mathbf{6 5} 064512$

[13]Ludwig F and Drung D 1999 Low-frequency noise of improved direct-coupled high-T c superconducting quantum interference device magnetometers in ac and dc magnetic fields Appl. Phys. Lett. 75 2821-3

[14]Baziljevich M, Baruch-El E, Johansen T H and Yeshurun Y 2014 Dendritic instability in $\mathrm{YBa}_{2} \mathrm{Cu}_{3} \mathrm{O}_{7-\delta}$ films triggered by transient magnetic fields Appl. Phys. Lett.

105012602

[15] Song H, Hunte F and Schwartz J 2012 On the role of pre-existing defects and magnetic flux avalanches in the degradation of $\mathrm{YBa}_{2} \mathrm{Cu}_{3} \mathrm{O}_{7-\mathrm{x}}$ coated conductors by quenching Acta Mater. 60 6991-7000

[16]Carmo D, Colauto F, de Andrade A M H, Oliveira A A M, Ortiz W A and Johansen T H 2016 Controllable injector for local flux entry into superconducting films Supercond. Sci. Tech. 29095003

[17]Zhou D F, Ainslie M D, Srpcic J, Huang K Y, Shi Y H, Dennis A R, Cardwell D A, Durrell J H, Boll M and Filipenko M 2018 Exploiting flux jumps for pulsed field magnetisation Supercond. Sci. Tech. 31105005

[18]Ainslie M D, Zhou D, Fujishiro H, Takahashi K, Shi Y H and Durrell J H 2016 Flux jump-assisted pulsed field magnetisation of high-Jc bulk high-temperature superconductors Supercond. Sci. Tech. 29124004

[19]Fisher L M, Johansen T H, Bobyl A, Rakhmanov A L, Nesterov M L and Yampol'skii V A 2006 Low Temp. Phys., (Melville: Amer Inst Physics) pp 805-6

[20]Altinkok A, Kilic K, Olutas M and Kilic A 2014 Dynamic Instabilities and CurrentVoltage Curves of Polycrystalline Superconducting $\mathrm{Y}_{1} \mathrm{Ba}_{2} \mathrm{Cu}_{3} \mathrm{O}_{7-\mathrm{x}} / \mathrm{Ag}$ J. Supercond. Nov. Magn. 27 651-65

[21] Gao L, Xue Y Y, Meng R L and Chu C W 1994 Thermal-Instability, Magnetic-Field Shielding and Trapping in Single-Grain $\mathrm{YBa}_{2} \mathrm{Cu}_{3} \mathrm{O}_{7-D e l t a}$ Bulk Materials Appl. Phys. 
Lett. $64520-2$

[22] Johansen T H, Baziljevich M, Shantsev D V, Goa P E, Galperin Y M, Kang W N, Kim H J, Choi E M, Kim M S and Lee S I 2002 Dendritic magnetic instability in superconducting $\mathrm{MgB}_{2}$ films Europhys. Lett. 59 599-605

[23] Treiber S and Albrecht J 2010 The formation and propagation of flux avalanches in tailored $\mathrm{MgB}_{2}$ films New J. Phys. 12 1367-2630

[24]Sumption M D and Collings E W 2003 Stability and flux jumping of internal-Sn, $\mathrm{Nb}_{3} \mathrm{Sn}$ conductors (and a model system $\mathrm{MgB}_{2}$ ) IEEE Trans. Appl. Supercond. $133394-$ 7

[25] Ghosh A K, Cooley L D and Moodenbaugh A R 2005 Investigation of instability in high $\mathrm{J}_{\mathrm{c}} \mathrm{Nb}_{3} \mathrm{Sn}$ strands IEEE Trans. Appl. Supercond. 15 3360-3

[26]Rudnev I A, Antonenko S V, Shantsev D V, Johansen T H and Primenko A E 2003

Dendritic flux avalanches in superconducting $\mathrm{Nb}_{3} \mathrm{Sn}$ films Cryogenics 43 663-6

[27] Wimbush S C, Holzapfel B and Jooss C 2004 Observation of dendritic flux instabilities in $\mathrm{YNi}_{2} \mathrm{~B}_{2} \mathrm{C}$ thin films $J$. Appl. Phys. 96 3589-91

[28]Awad A A, Aliev F G, Ataklti G W, Silhanek A, Moshchalkov V V, Galperin Y M and Vinokur V 2011 Flux avalanches triggered by microwave depinning of magnetic vortices in $\mathrm{Pb}$ superconducting films Phys. Rev. B 84 1098-0121

[29]Hebert S, Van Look L, Weckhuysen L and Moshchalkov V V 2003 Vortex avalanches in a Pb film with a square antidot array Phys. Rev. B 67 1098-0121

[30]Menghini M, Van de Vondel J, Gheorghe D G, Wijngaarden R J and Moshchalkov V V 2007 Asymmetry reversal of thermomagnetic avalanches in $\mathrm{Pb}$ films with a ratchet pinning potential Phys. Rev. B 76 1098-0121

[31] Mikheenko P, Qviller A J, Vestgarden J I, Chaudhuri S, Maasilta I J, Galperin Y M and Johansen T H 2013 Nanosecond voltage pulses from dendritic flux avalanches in superconducting NbN films Appl. Phys. Lett. 102 0003-6951

[32]Denisov D V, Rakhmanov A L, Shantsev D V, Galperin Y M and Johansen T H 2006 Dendritic and uniform flux jumps in superconducting films Phys. Rev. B 73

[33]Dvash E E, Shapiro I and Shapiro B Y 2008 Flux pattern instability in a strongly anisotropic type-II superconducting slab Europhys. Lett. 83 67005-5

[34]Dvash E E, Shapiro I and Shapiro B Y 2009 Dendritic instability of the magnetic flux in thermally anisotropic type-II superconductors Phys. Rev. B 80 1098-0121

[35]Rakhmanov A L, Shantsev D V, Galperin Y M and Johansen T H 2004 Finger patterns produced by thermomagnetic instability in superconductors Phys. Rev. B 70 224502

[36] Aranson I S, Gurevich A, Welling M S, Wijngaarden R J, Vlasko-Vlasov V K, Vinokur V M and Welp U 2005 Dendritic flux avalanches and nonlocal electrodynamics in thin superconducting films Phys. Rev. Lett. 94037002

[37]Aranson I S, Kopnin N B and Vinokur V M 2001 Dynamics of vortex nucleation by rapid thermal quench Phys. Rev. B 63 184501-12

[38]Aranson I, Gurevich A and Vinokur V 2001 Vortex avalanches and magnetic flux fragmentation in superconductors Phys. Rev. Lett. 87067003

[39]Zhou Y-H and Yang X 2006 Numerical simulations of thermomagnetic instability in high- $\mathrm{T}_{\mathrm{c}}$ superconductors: Dependence on sweep rate and ambient temperature Phys. 
Rev. B 74054507

[40]Xia J, Li M and Zhou Y 2017 Numerical investigations on the characteristics of thermomagnetic instability in $\mathrm{MgB}_{2}$ bulks Supercond. Sci. Tech. 30075004

[41] Vestgården J I, Mikheenko P, Galperin Y M and Johansen T H 2013 Nonlocal electrodynamics of normal and superconducting films New J. Phys. 15 1367-2630

[42] Vestgården J I, Shantsev D V, Galperin Y M and Johansen T H 2011 Dynamics and morphology of dendritic flux avalanches in superconducting films Phys. Rev. B 84 1098-0121

[43] Vestgården J I, Shantsev D V, Galperin Y M and Johansen T H 2012 Lightning in superconductors Sci. Rep. 2886

[44] Vestgården J I, Shantsev D V, Galperin Y M and Johansen T H 2013 The diversity of flux avalanche patterns in superconducting films Supercond. Sci. Tech. 26 0953-2048 [45] Vestgården J I, Shantsev D V, Galperin Y M and Johansen T H 2011 Dynamics and morphology of dendritic flux avalanches in superconducting films Phys. Rev. B 84 054537

[46]Jing Z, Yong H D and Zhou Y H 2016 Influences of non-uniformities and anisotropies on the flux avalanche behaviors of type-II superconducting films Supercond. Sci. Tech. 29 1-13

[47] Jing Z, Yong H D and Zhou Y H 2017 Numerical simulation on the flux avalanche behaviors of microstructured superconducting thin films J. Appl. Phys. 121023902

[48]Prigozhin L and Sokolovsky V 2018 Fast Fourier transform-based solution of 2D and 3D magnetization problems in type-II superconductivity Supercond. Sci. Tech. 31 055018

[49] Motta M, Colauto F, Zadorosny R, Johansen T H, Dinner R B, Blamire M G, Ataklti G W, Moshchalkov V V, Silhanek A V and Ortiz W A 2011 Visualizing the ac magnetic susceptibility of superconducting films via magneto-optical imaging Phys. Rev. B 84 214529

[50]Motta M, Colauto F, Johansen T H, Dinner R B, Blamire M G, Ataklti G W, Moshchalkov V V, Silhanek A V and Ortiz W A 2012 Flux avalanches triggered by AC magnetic fields in superconducting thin films Physica C 479 134-6

[51] Vestgarden J I, Galperin Y M and Johansen T H 2012 Dendritic flux avalanches in rectangular superconducting films - Numerical simulations Physica C 479 92-4

[52]Motta M, Colauto F, Vestgården J I, Fritzsche J, Timmermans M, Cuppens J, Attanasio C, Cirillo C, Moshchalkov V V, Van de Vondel J, Johansen T H, Ortiz W A and Silhanek A V 2014 Controllable morphology of flux avalanches in microstructured superconductors Phys. Rev. B 89134508

[53]Kim Y, Hempstead C and Strnad A 1963 Magnetization and critical supercurrents Phys. Rev. 129528

[54] Vestgarden J I, Mikheenko P, Galperin Y M and Johansen T H 2013 Nonlocal electrodynamics of normal and superconducting films New J. Phys. 15093001

[55] Vestgarden J I, Galperin Y M and Johansen T H 2016 Oscillatory regimes of the thermomagnetic instability in superconducting films Phys. Rev. B 93174511

[56]Denisov D V, Shantsev D V, Galperin Y M, Choi E M, Lee H S, Lee S I, Bobyl A V, Goa P E, Olsen A A and Johansen T H 2006 Onset of dendritic flux avalanches in 
superconducting films Phys. Rev. Lett. 97077002

[57]Welling M S, Westerwaal R J, Lohstroh W and Wijngaarden R J 2004 Huge compact flux avalanches in superconducting $\mathrm{Nb}$ thin films Physica $C \mathbf{4 1 1}$ 11-7

[58]Il'in K, Rall D, Siegel M, Engel A, Schilling A, Semenov A and Huebers H W 2010 Influence of thickness, width and temperature on critical current density of $\mathrm{Nb}$ thin film structures Physica C 470 953-6

[59]Colauto F, Patiño E J, Aprilli M and Ortiz W A 2009 The role of demagnetizing factors in the occurrence of vortex avalanches in Nb thin films J. Phys. Conf. Ser. 150 052038

[60]Embon L, Anahory Y, Jelic Z L, Lachman E O, Myasoedov Y, Huber M E, Mikitik G P, Silhanek A V, Milosevic M V, Gurevich A and Zeldov E 2017 Imaging of superfast dynamics and flow instabilities of superconducting vortices Nat. Commun. 885

(a)

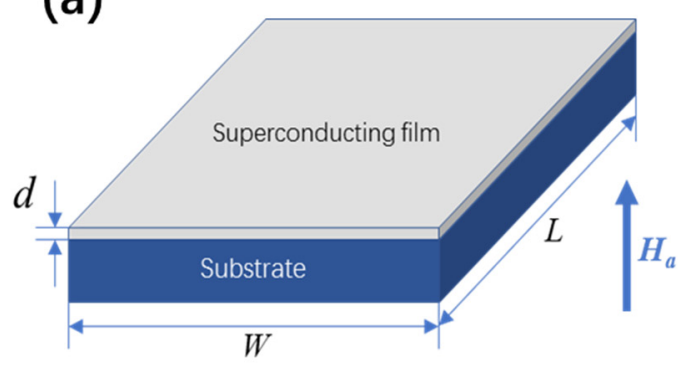

(b)

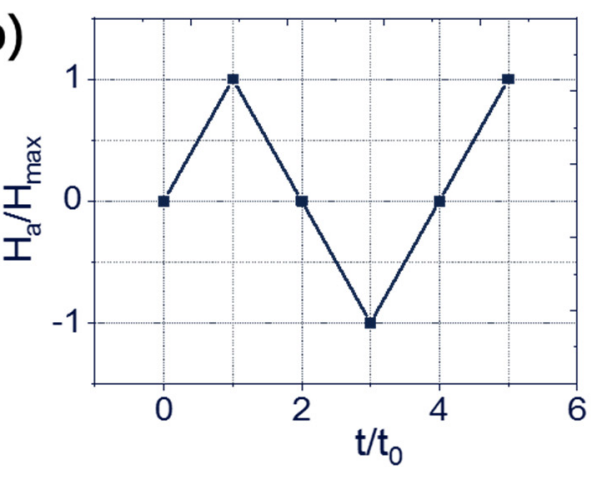

Figure 1. (a) sketch of the modeled superconducting film-substrate system and (b) waveform of the transient AC magnetic field. 


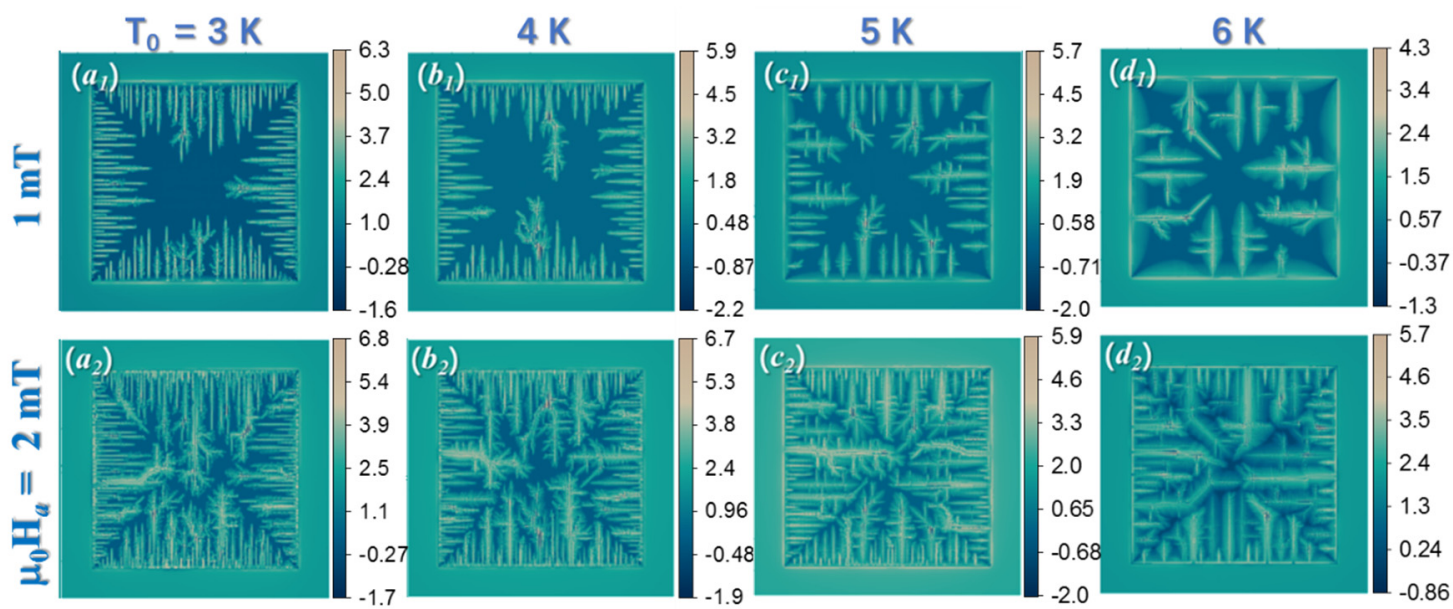

Figure 2. Flux avalanche patterns of the SC film subjected to an external magnetic field ramped from 0 to $2 \mathrm{mT}$ at a rate of $1000 \mathrm{~T} / \mathrm{s}(\mathrm{f}=125 \mathrm{kHz})$, at $3 \mathrm{~K}, 4 \mathrm{~K}, 5 \mathrm{~K}$ and $6 \mathrm{~K}$ ambient temperatures, respectively. $\left(\mathrm{a}_{1}\right)-\left(\mathrm{d}_{1}\right)$ and $\left(\mathrm{a}_{2}\right)-\left(\mathrm{d}_{2}\right)$ are avalanche patterns when the external field ramped from zero to $1 \mathrm{mT}$ and $2 \mathrm{mT}$, respectively. 


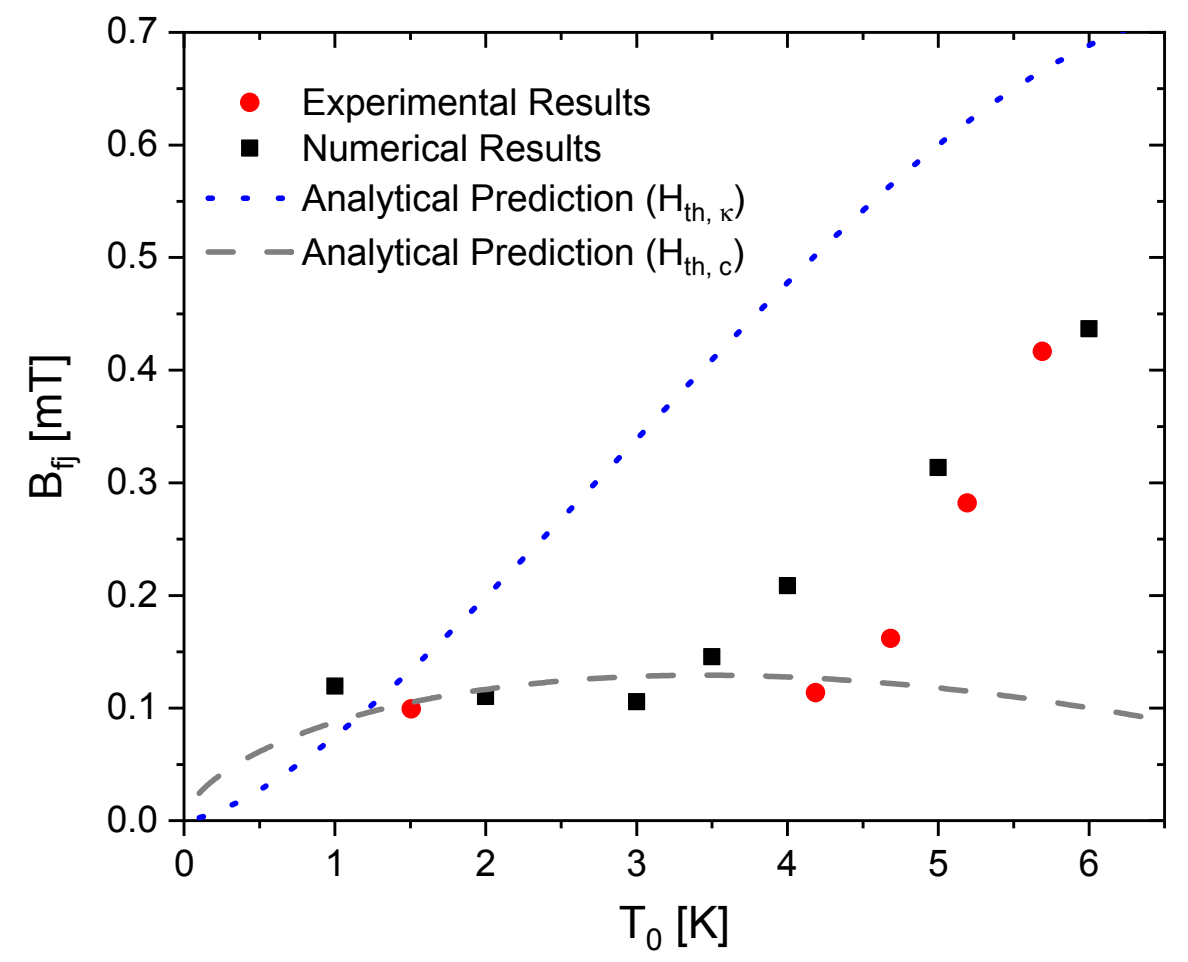

Figure 3 Temperature dependence of the threshold magnetic field for the $\mathrm{Nb}$ thin film with thickness $d=50 \mathrm{~nm}$ and the external magnetic field ramp rate $\mu_{0} \dot{H}_{a}=1000 \mathrm{~T} / \mathrm{s} \quad$ (f $=125 \mathrm{kHz})$. 


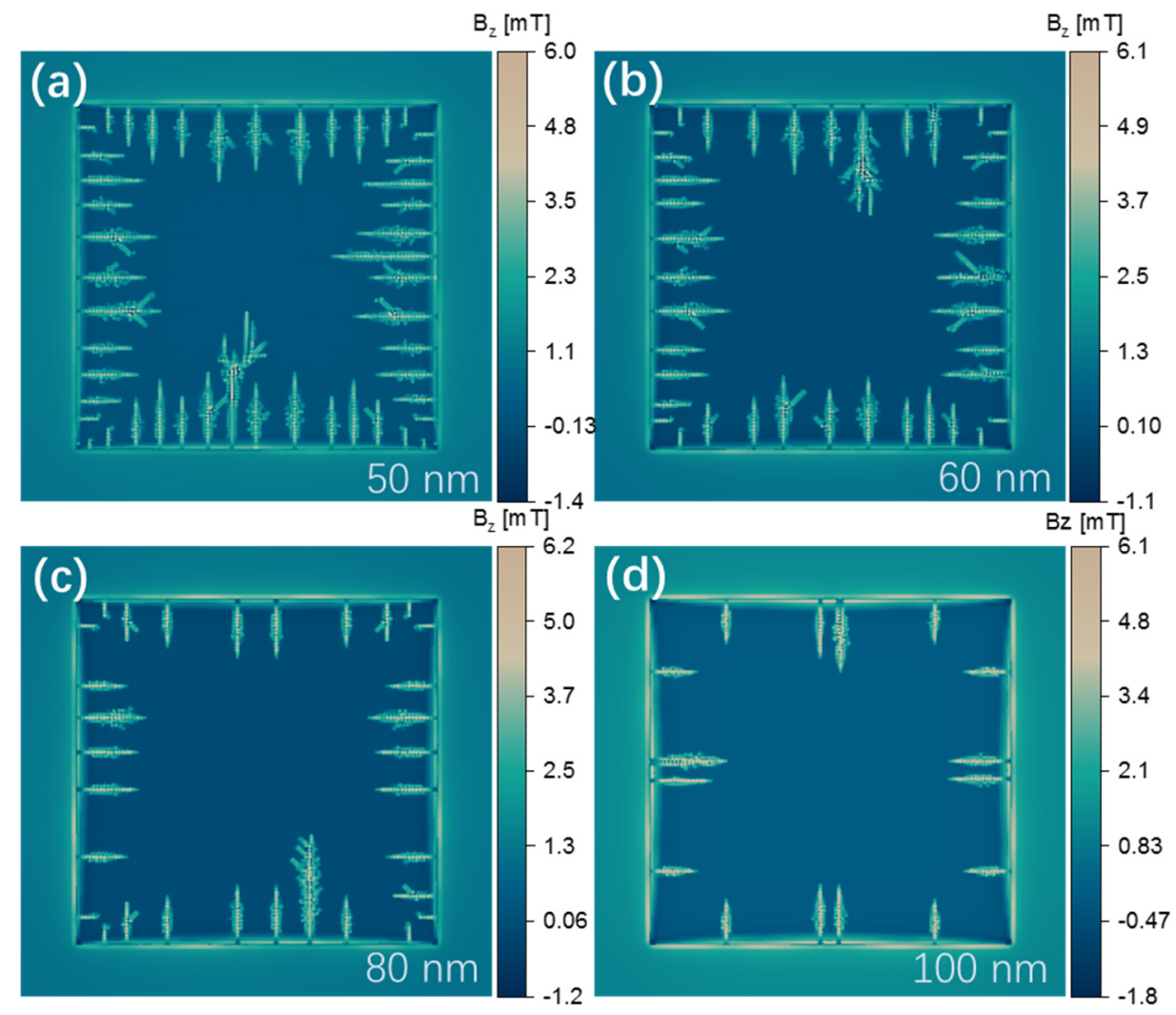

Figure 4. Flux avalanche patterns in SC films of different thicknesses subjected to an external magnetic field ramped from 0 to $1 \mathrm{mT}$ at a ramp rate of $50 \mathrm{~T} / \mathrm{s}(\mathrm{f}=6.25 \mathrm{kHz})$, at $3 \mathrm{~K}$ ambient temperature. 


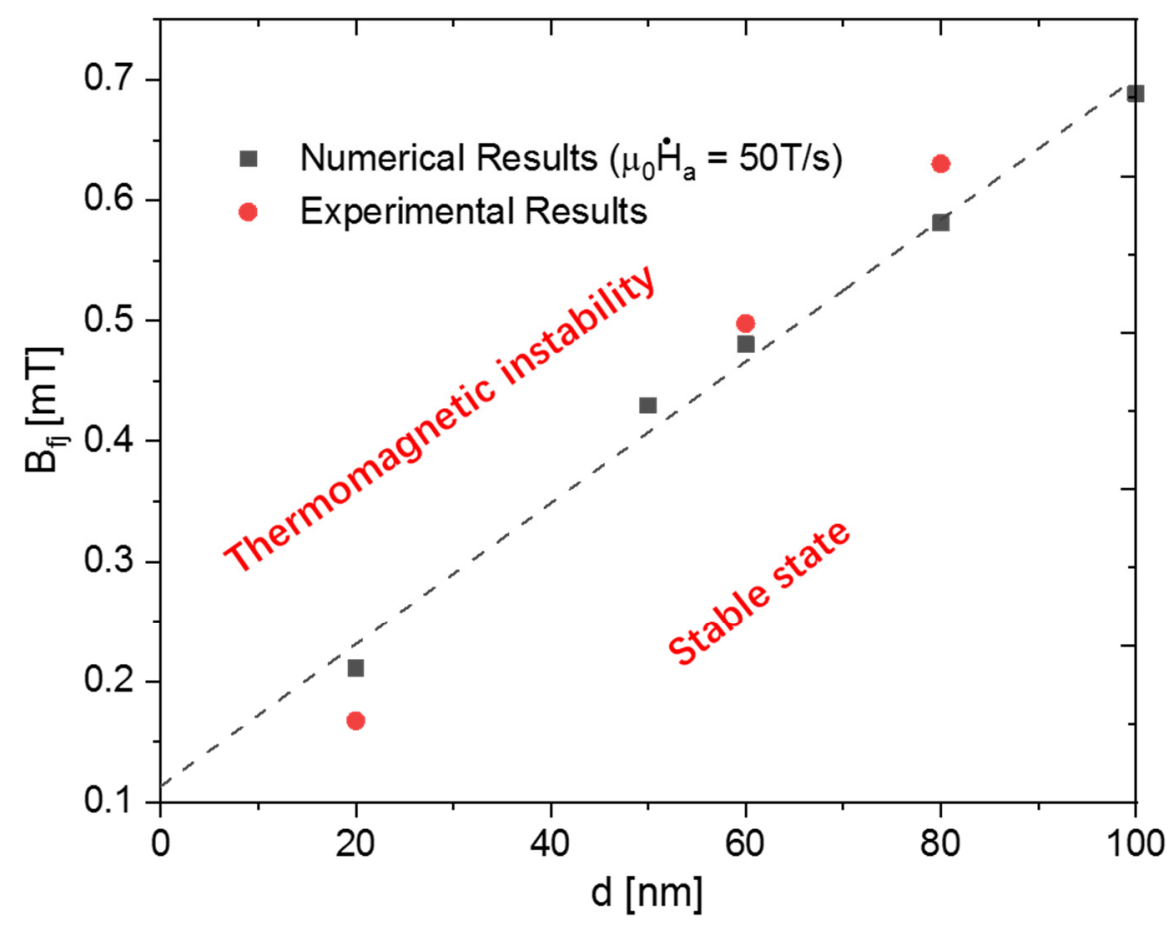

Figure 5 Thickness dependence of the threshold magnetic field for the $\mathrm{Nb}$ thin film under an increasing magnetic field of ramp rate $\mu_{0} \dot{H}_{a}=1000 \mathrm{~T} / \mathrm{s} \quad(\mathrm{f}=125 \mathrm{kHz})$, at 3 $\mathrm{K}$ ambient temperature. 

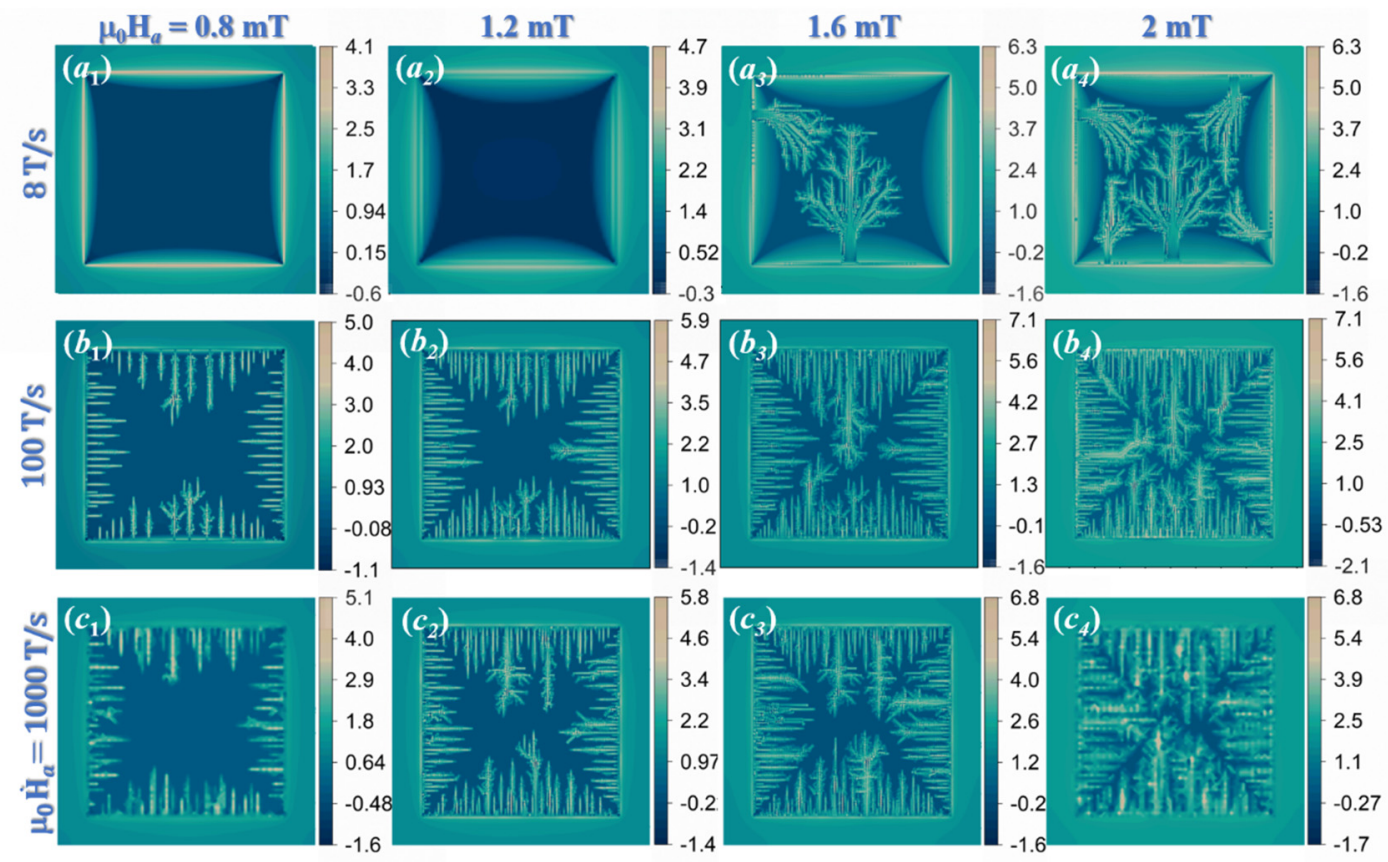

Figure 6 Flux avalanche patterns in the $\mathrm{Nb}$ film of thickness $d=50 \mathrm{~nm}$, subjected to an external magnetic field ramped from 0 to $2 \mathrm{mT}$ with different ramp rates, at the ambient temperature $T_{0}=3 \mathrm{~K}$. ( (a1) $-\left(\mathrm{c}_{1}\right),\left(\mathrm{a}_{2}\right)-\left(\mathrm{c}_{2}\right),\left(\mathrm{a}_{3}\right)-\left(\mathrm{c}_{3}\right)$ and $\left(\mathrm{a}_{4}\right)-\left(\mathrm{c}_{4}\right)$ are avalanche patterns when the external field ramped from zero to $0.8 \mathrm{mT}, 1.2 \mathrm{mT}, 1.6 \mathrm{mT}$ and 2.0 $\mathrm{mT}$, respectively. 


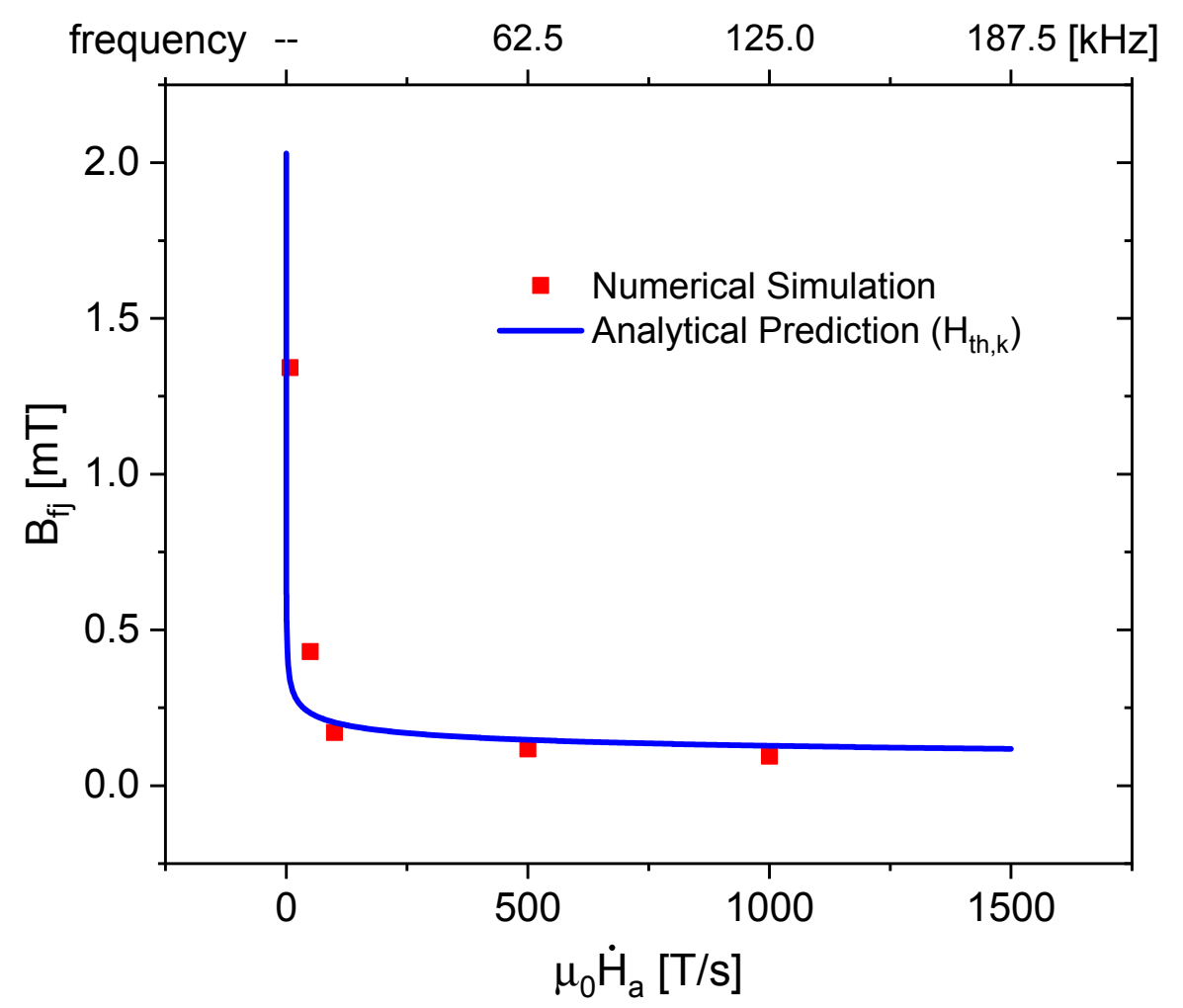

Figure 7 Threshold magnetic field dependence on the external magnetic field ramp rate, for the $\mathrm{Nb}$ thin film of thickness $50 \mathrm{~nm}$ at $3 \mathrm{~K}$ ambient temperature. 

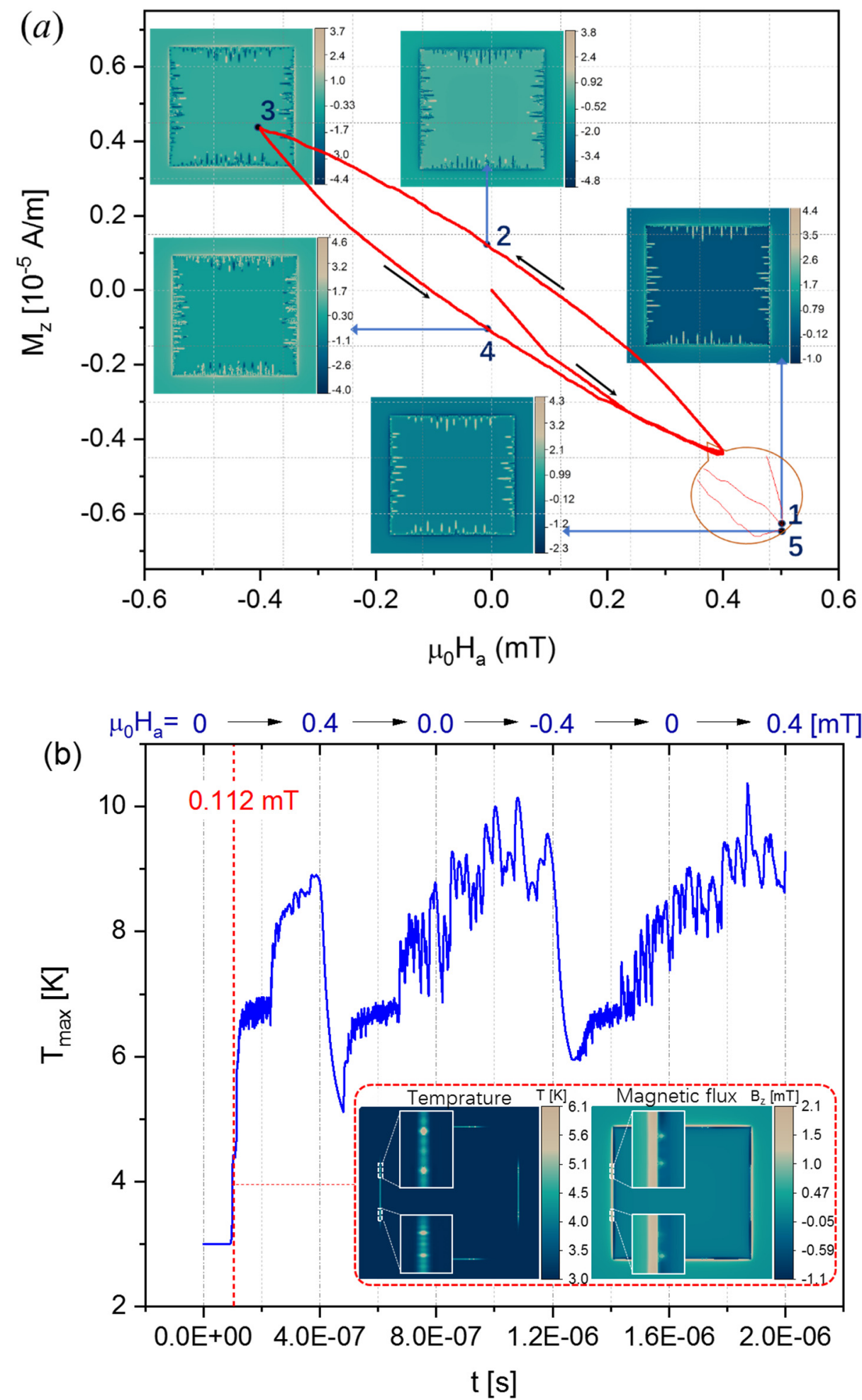

Figure 8 (a) Average magnetization jump curve and (b) maximum temperature in the $50 \mathrm{~nm}$ thick $\mathrm{Nb}$ thin film exposed to a transient $\mathrm{AC}$ magnetic field ramped from $0 \mathrm{mT}$ $\rightarrow+0.4 \mathrm{mT} \rightarrow 0 \mathrm{mT} \rightarrow-0.4 \mathrm{mT} \rightarrow 0 \mathrm{mT} \rightarrow+0.4 \mathrm{mT}$ at a rate of $1000 \mathrm{~T} / \mathrm{s} \quad(\mathrm{f}=125$ $\mathrm{kHz}$ ), at $3 \mathrm{~K}$ ambient temperature. The inset images 1-5 in figure 8(a) show the flux avalanche patterns in the SC film when the external magnetic field is ramped to the corresponding points indicated by the black dots. 


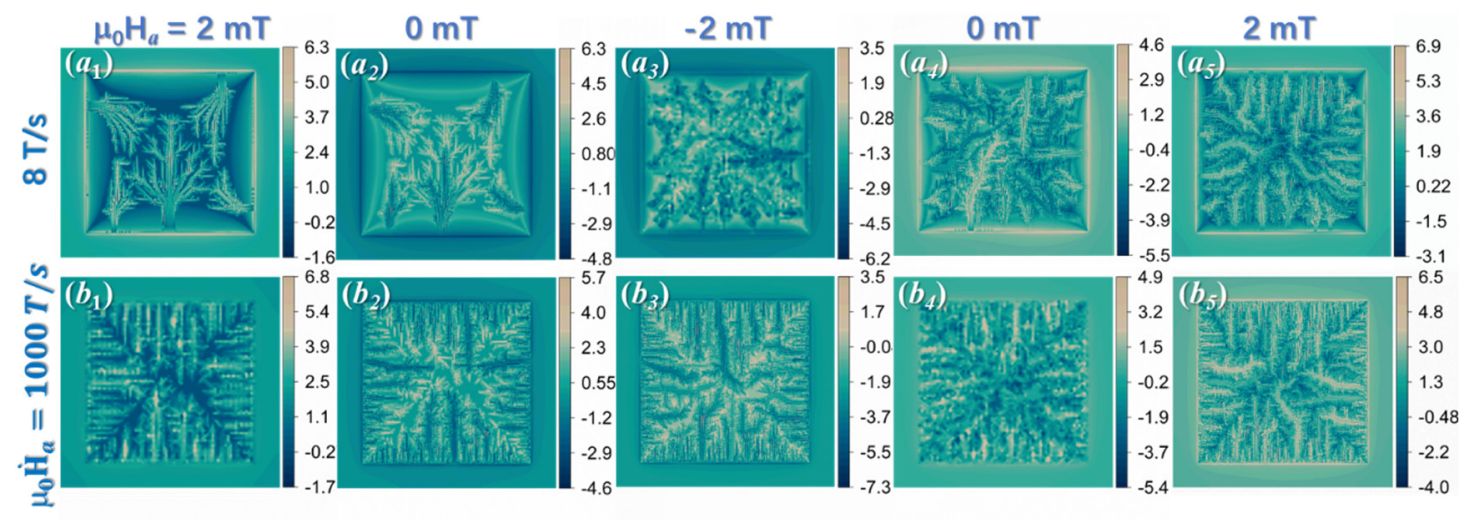

Figure 9. Magnetic flux avalanche patterns in the $\mathrm{Nb}$ film exposed to $\mathrm{AC}$ magnetic fields with ramp rates of $8 \mathrm{~T} / \mathrm{s}$ and $1000 \mathrm{~T} / \mathrm{s}$ respectively, at the ambient temperature $T_{0}$ $=3 \mathrm{~K} .\left(\mathrm{a}_{1}\right)-\left(\mathrm{a}_{5}\right)$ and $\left(\mathrm{b}_{1}\right)-\left(\mathrm{b}_{5}\right)$ are avalanche patterns when the magnetic field ramped from $0 \mathrm{mT} \rightarrow+2 \mathrm{mT} \rightarrow 0 \mathrm{mT} \rightarrow-2 \mathrm{mT} \rightarrow 0 \mathrm{mT} \rightarrow+2 \mathrm{mT}$ at the rates of $8 \mathrm{~T} / \mathrm{s}$ and $1000 \mathrm{~T} / \mathrm{s}$, respectively. 


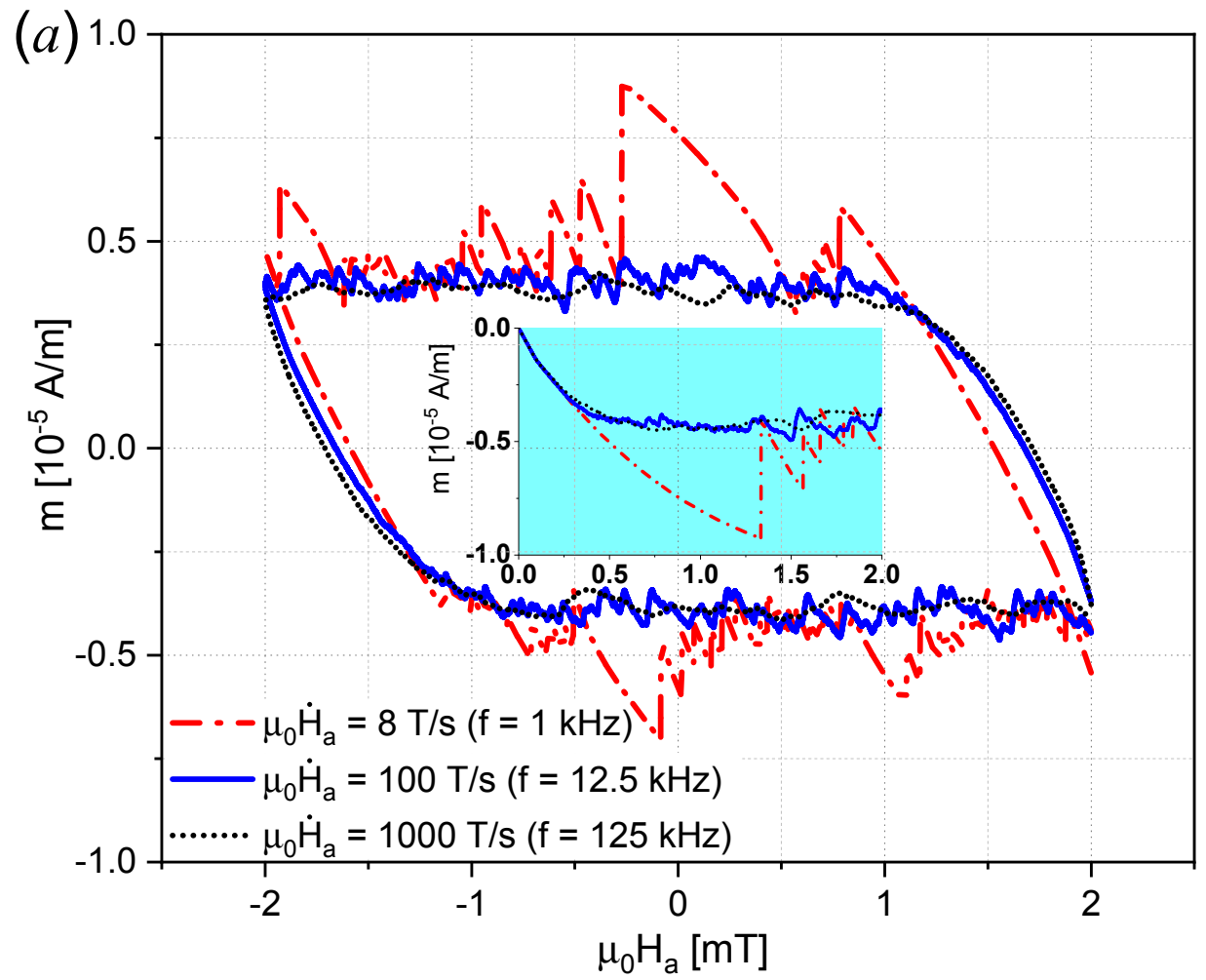

(b) $\mu_{0} \mathrm{H}_{\mathrm{a}}=\mathrm{O} \longrightarrow+2 \longrightarrow 0 \longrightarrow-2 \longrightarrow 0 \longrightarrow+2[\mathrm{mT}]$
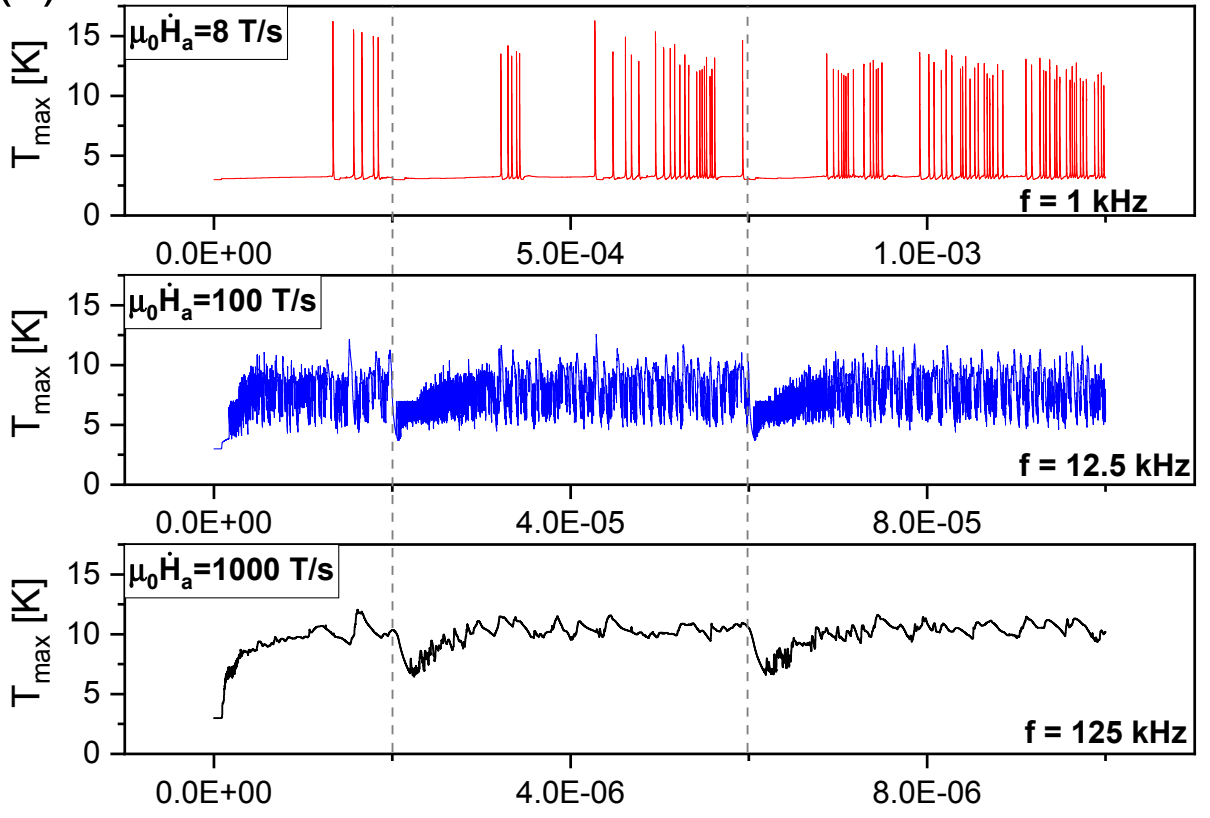

$\mathrm{t}[\mathrm{s}]$

Figure 10 (a) Average magnetization jump curves and (b) maximum temperature of the $\mathrm{Nb}$ thin film (with $50 \mathrm{~nm}$ thickness) under a transient $\mathrm{AC}$ external magnetic field ramped from $0 \mathrm{mT} \rightarrow+2 \mathrm{mT} \rightarrow 0 \mathrm{mT} \rightarrow-2 \mathrm{mT} \rightarrow 0 \mathrm{mT} \rightarrow+2 \mathrm{mT}$ at different ramp rates, at $3 \mathrm{~K}$ ambient temperature. 